\title{
Índice Socioeconômico (ISE) utilizado na mensuração do desenvolvimento regional no estado de Santa Catarina
}

Vinicius Tischer ${ }^{1}$

\begin{abstract}
Resumo
O entendimento de fenômenos sociais é fundamental para delinear políticas públicas e subsidiar tomadas de decisões, e a sua mensuração é determinante para desenvolver estratégias visando elevar as condições sociais da população. Com isso, o trabalho teve por objetivo desenvolver um Índice Socioeconômico (ISE) tendo por estudo de caso o estado de Santa Catarina. Foram utilizados dados exclusivos do Censo Demográfico de 2010, utilizando-se quatro temáticas de abordagem: autonomia de renda, desenvolvimento humano, equidade e infraestrutura urbana. Foi obtido o ISE, em nível de setor censitário para todo o estado, o qual variou de $-4,77$ a 2,42, sendo identificados, com base no índice desenvolvido, setores, municípios e regiões com melhores e piores condições socioeconômicas.
\end{abstract}

Palavras-chave: Indicadores socioeconômicos. Índice socioeconômico. Sistema de Informação Geográfica. Censo demográfico.

\begin{abstract}
The understanding of social phenomena is fundamental to delineate public policies and subsidize decision making and its measurement is determinant to develop strategies to raise the social conditions of the population. The purpose of this study was to develop a Socioeconomic Index (ISE), based on a case study of the State of Santa Catarina. It was only used data from the 2010 demographic census, using four thematic approaches: income autonomy, human development, equity and urban infrastructure. The ISE was obtained at the level of the census sector for the whole state, which varied from $-4,77$ to 2,42, and identified sectors, municipalities and regions with better and worse socioeconomic conditions based on the developed index.
\end{abstract}

Keywords: Socioeconomic. Socioeconomic indicators. Socioeconomic index. Geographic Information System. Demographic census.

\section{Introdução}

Fenômenos sociais influenciam diretamente na qualidade de vida e no desenvolvimento humano, econômico e político. O seu entendimento é fundamental para delinear políticas públicas e subsidiar tomadas de decisões. Assim, a mensuração de parâmetros objetivos são determinantes para o desenvolvimento de estratégias e intervenções necessárias para elevar as condições sociais da população.

\footnotetext{
${ }^{1}$ Doutorando do Programa de Pós-Graduação em Ciências e Tecnologia Ambiental da Universidade do Vale do Itajaí, SC, Brasil. tischer@edu.univali.br
} 
Nesse sentido, para mensurar estas variáveis, indicadores conhecidos por "indicadores sociais" ou "indicadores socioeconômicos" têm papel preponderante (SOLIGO, 2012). Para Bauer (1966), estatísticas, séries estatísticas e todas as outras formas de evidência permitem avaliar o estado atual e as tendências em relação a valores e objetivos, avaliar programas específicos e determinar seu impacto.

Segundo Jannuzzi (2009), indicadores sociais referem-se a medidas, quantitativas em geral, que denotam significado social substantivo. São um recurso metodológico utilizado para substituir, quantificar ou operacionalizar um conceito abstrato, empiricamente referido, que informa algo sobre um aspecto da realidade social ou sobre mudanças que estão em curso.

Indicadores têm sido muito utilizados para diagnosticar variados fenômenos de interesse, por meio, geralmente, de conjunto denominado sistema de indicadores. Segundo Tischer e Polette (2016), um sistema de indicador refere-se a um conjunto de indicadores concernentes a um mesmo tema, em que cada um dos indicadores é suplementar ao outro e explica determinado fenômeno dentro de um contexto. Para o Instituto Brasileiro de Geografia e Estatística (IBGE, 2015), um conjunto de indicadores sociais sistematiza informações sobre a realidade social do país, a partir de temas de grande relevância, permitindo a construção de um quadro abrangente e integrado, considerando as distintas dimensões que envolvem a elaboração de um sistema de indicadores sociais.

Estudo do IBGE (2015b) ressalta que um conjunto de indicadores sociais contribui para a compreensão das modificações nos perfis demográfico, social e econômico da população, possibilitando, assim, o monitoramento de políticas sociais e a disseminação de informações relevantes para toda a sociedade brasileira. Jannuzzi (2009) complementa que os indicadores auxiliam no planejamento e formulação de políticas públicas, possibilitando o monitoramento das condições de vida e bem-estar da população por parte do poder público e da sociedade civil, permitindo um aprofundamento da investigação científica sobre diferentes fenômenos sociais.

O censo demográfico é a mais complexa operação estatística realizada por um país, sendo investigadas características de toda população e domicílios do território nacional. No 
Brasil, o acesso à base de dados censitários é fornecido pelo IBGE, sendo disponibilizados dados e metadados diversificados em sua plataforma eletrônica.

Nos últimos anos, estes dados estão sendo associados a objetos com identificador geográfico, que permite o seu georreferenciamento para uso em Sistema de Informação Geográfica (SIG), sendo que, para o Censo Demográfico 2010, foi produzido o primeiro arquivo com dados em nível de setor censitário, contendo cerca de 3000 variáveis (IBGE, 2011). Isso permite aumentar o ganho de conhecimento e as análises possíveis, ampliando as possibilidades de disseminação de informações à sociedade. Dependendo do dado, há uma elevada desagregação geográfica, desde a unidade mais elementar do censo (setor censitário) até outras, como bairros, distritos, município, região metropolitana, etc. O setor censitário é a menor unidade territorial e de controle cadastral da coleta, formada por área contínua, integralmente contida em área urbana ou rural, com dimensão adequada à operação de pesquisas, com cobertura ao longo de todo o país (IBGE, 2011).

A base territorial do Censo Demográfico 2010 foi elaborada de forma a integrar a representação espacial das áreas urbana e rural do território nacional em um ambiente de banco de dados geoespaciais, utilizando insumos e recursos de tecnologia da informação (IBGE, 2011). A adoção de medidas passíveis de comparabilidade, ao longo de todo território nacional, por meio de dados provenientes de censos demográficos, torna-se cada vez mais desejável para o planejamento público, permitindo um acesso imediato ao banco de dados e fazendo uso das informações levantadas.

E ainda, é importante que indicadores empregados correspondam a propriedades de desempenho, para que sejam o mais robusto possível em sua utilização. Em relação à análise das propriedades de desempenho de indicadores, Tischer (2016) afirma que um indicador pode ser importante para a explicação de determinado fenômeno: todavia, sua obtenção pode não ser viabilizada, não possuindo uma periodicidade, ficando, por exemplo, condicionado a subsídios financeiros para ser desenvolvido.

Nesse sentido, informações do censo possuem importância, por suprir essas deficiências de geração de dados nos objetivos e indicadores buscados pelo levantamento, ressaltandose ainda o ganho para análises geoespaciais, possibilitando que o planejamento seja subsidiado, permitindo determinar prioridades de forma prática, que podem fornecer 
ganhos de gestão pública de forma eficiente e eficaz. Ressalta-se que, mesmo com essa ampla base de dados do IBGE, as informações são subutilizadas, seja por questões de desconhecimento, ou por falta de perícia dos planejadores públicos na utilização do sistema.

A avaliação das condições socioeconômicas vem sendo tratada por meio de estudos de Nível Socioeconômico (NSE). Alves e Soares (2009) citam que o NSE é uma variável latente (não diretamente observada), cuja medida é feita pela agregação de informações sobre a educação, a ocupação e a riqueza ou rendimento dos indivíduos. Apesar da existência de inúmeros estudos acerca do nível socioeconômico enquanto variável explicativa de diversos fenômenos sociais, não há um consenso na literatura sobre sua conceituação, bem como sobre como medi-lo nas pesquisas empíricas.

Associado a isso, diversos estudos que tratam da situação socioeconômica não conceituam ou delimitam esse conceito enquanto variável de uso prático. Segundo glossário da JICZM (2017), o termo socioeconômico diz respeito a variáveis reconhecidas por órgãos oficiais (IBGE, PNUD, OMS, FAO, UNESCO) como descritores das condições sociais reinantes em uma população: taxas de natalidade, mortalidade, escolaridade, fertilidade, alunos matriculados em escolas, renda, incidência de infecções, etc.

Cabe destacar que, na prática, a utilização de sistemas de indicadores, usualmente os indicadores proxy, que refletem um determinado parâmetro de estudo, sem, necessariamente, utilizar-se de medidas diretas do fenômeno, como, por exemplo, indicadores relacionados a condições de saneamento básico (esgoto a céu aberto ou coleta de lixo, por exemplo) podem servir para o entendimento de deficiências econômicas de determinado domicílio (grupo), ou mesmo correlacionar-se à esperança de vida ao nascer e à taxa de mortalidade infantil, conforme exemplificado pela matriz de relacionamento de indicadores do IBGE (IBGE, 2015).

No Brasil, a criação de índices de avaliação está geralmente relacionada ao desenvolvimento de índices relativos à exclusão social, e se utiliza de dados socioeconômicos para a unificação de medidas que permitam localizar áreas socialmente mais vulneráveis. Segundo Singer (2003), a exclusão social refere-se a um total de exclusões, habitualmente muito inter-relacionadas, tais como pessoas expulsas do mercado de trabalho formal, ou do mercado da residência formal (em contraste com o informal, formado por 
cortiços e favelas), ou da escola, as quais ficam em desvantagem na competição por novas oportunidades, sendo suscetíveis a prováveis novas exclusões.

Estudo da Organização Mundial da Saúde reuniu diversos conceitos de exclusão social (MATHIESON et al., 2008), onde, por exemplo, Barnes (2005) afirma que exclusão social se refere ao processo dinâmico e multidimensional de ser impedido, total ou parcialmente, de sistemas econômico, social e cultural, os quais determinam a integração da pessoa na sociedade. Já para Byrne (1999), a exclusão social exclui o indivíduo de várias formas combinadas (participação na tomada de decisões e processos políticos, acesso ao emprego e recursos materiais e integração em processos culturais comuns), que, quando combinadas, criam formas agudas de exclusão, que encontram uma manifestação espacial em bairros particulares.

Lemos (2007) destaca que uma das principais variáveis relacionadas à exclusão social é a pobreza, que consiste em uma condição de privação das condições materiais, em nível de satisfação minimamente aceitável, das necessidades humanas (alimentação, serviços de saúde, educação) e de serviços essenciais, como saneamento básico, etc.

Nesse sentido, Sposati (1996) desenvolveu uma metodologia para a determinação do Índice de Exclusão/Inclusão Social (IEX), usando como estudo de caso o município de São Paulo. O método emprega indicadores socioeconômicos integrados à SIG (utilizando como unidades territoriais os distritos) e produz índices territoriais que hierarquizam regiões de uma cidade quanto ao grau de exclusão/inclusão social. O Mapa da Exclusão/Inclusão Social parte da releitura e da inter-relação existente esses dados censitários e mais outros dados produzidos por órgãos municipais sobre toda a cidade (SPOSATI, 2000).

Este estudo serviu de fundamentação para diversas outras pesquisas. Genovez et al. (2001) adaptou o método de Sposati (1996), passando a utilizar setores censitários como unidades de análise, ao invés dos distritos, com objetivo de dotar a análise de maior resolução espacial. Para o estudo de caso, foram utilizados dados do censo demográfico do ano de 1991, contendo um total de 291 variáveis, relativas a 356 setores censitários referentes à área urbana do município de São José dos Campos - SP.

Silva Júnior (2007) avaliou as condições socioeconômicas de Itajaí (SC), chegando a resultados contundentes acerca das condições de desigualdade no município, caracterizando 
que quase $60 \%$ da população encontram-se em situação de exclusão territorial, com carências nos mais diversos parâmetros avaliados. Para a composição do índice de exclusão social, o autor utilizou um método adaptado de Sposati (1996) em associação com dados gerados pelo IBGE, Prefeitura Municipal e Instituto Pólis. No trabalho, a integração geográfica utilizou os setores censitários agregados a Unidades Locais de Gestão (ULG), delimitadas pela Prefeitura Municipal, que são espaços demarcados segundo as identidades grupais em relação aos espaços de vivência cotidiana, e a união destas foram unidades espaciais de gestão (UEG).

Nascimento e Matias (2008) avaliaram o índice de exclusão social para o município de Ponta Grossa/PR utilizando a fórmula proposta por Genovez (2005) para o cálculo dos índices, a qual garante uma maior precisão tanto para os indicadores das variáveis, como, e principalmente, para os indicadores compostos e índices finais. Com isso, realizaram uma comparação entre o índice gerado e os índices desenvolvidos por Sposati (1996) e Genovez et al. (2001), permitindo concluir que o método aperfeiçoado trouxe ganhos para uma identificação mais precisa das localidades em situações de exclusão social, além de exibir, mais detalhadamente, as condições desiguais de vida da população nas diferentes porções do espaço intraurbano, revelando uma cidade mais estratificada do que aquela retratada pelas outras duas metodologias.

A partir disso, importantes conceitos-chave podem ser considerados para a definição dos temas abordados: equidade, autonomia de renda, desenvolvimento humano e qualidade de vida, definidos por Sposati (1996) e Genovez et al. (2001) como:

- Equidade: efetivação da igualdade e do acesso aos direitos da população, possibilidade de a manifestação das diferenças serem respeitadas sem discriminação. Condição que favorece o combate à subordinação e ao preconceito em relação às diferenças entre homem e mulher.

- Autonomia de renda: compreendida como a capacidade do cidadão de suprir suas necessidades vitais, culturais, políticas e sociais, sob as condições de respeito às ideias individuais e coletivas, relacionando-se com o mercado, não importando apenas as responsabilidades do indivíduo, mas também as do Estado. 
- Desenvolvimento humano: possibilidade de os cidadãos desenvolverem seu potencial intelectual com menor grau de privação, ou seja, usufruir coletivamente do mais alto grau de capacidade humana.

- Qualidade de vida: refere-se à democratização dos acessos às condições de preservação do homem, da natureza e do meio ambiente, o que implica numa melhor redistribuição aos cidadãos da riqueza social e tecnológica, bem como redução da degradação e da precariedade ambiental.

$\mathrm{Na}$ análise dos autores citados, o tema qualidade de vida foi aferido, utilizando-se indicadores que remetem à infraestrutura urbana, os quais, independentemente da nomenclatura, permitem representar importantes características básicas, necessárias para a dignidade habitacional e de acesso aos serviços públicos no contexto urbano.

Neste sentido, o principal objetivo do trabalho foi desenvolver um Índice Socioeconômico (ISE) por meio de dados do censo do IBGE, utilizando nível de desagregação espacial para setores censitários. O objetivo secundário da pesquisa foi demonstrar as aplicações do SIG para as diferentes áreas do conhecimento, além de ampliar a utilização do extenso banco de dados do IBGE, onde sua exploração intensiva tem sido pouco empregada na governança pública.

O índice proposto não foi denominado explicitamente como exclusão, uma vez que permite uma gama maior de aplicações, sendo possível sua desagregação em atenção a critério específico dentro da composição do índice. Além disso, não aponta necessariamente para localidades/comunidades excluídas, sendo apenas, em média, um indicativo de condições melhores ou mais precárias em determinado setor do município.

\section{Metodologia}

Com base nas classes temáticas descritas anteriormente: equidade, autonomia de renda, desenvolvimento humano e qualidade de vida (substituído por infraestrutura urbana), foram definidos quais seriam os indicadores mais apropriados para representar estas classes, mantendo a representatividade dos indicadores levantados e, ao mesmo 
tempo, uma exequibilidade do sistema de indicadores. Os indicadores utilizados foram adaptados de Sposati (1996) e Genovez et al. (2001), sendo incluídos indicadores relacionados às infraestruturas de saneamento e urbana (características do entorno do domicílio). Ainda, foram eliminados indicadores similares, com informações semelhantes em outros indicadores, para que não fossem ocasionadas redundâncias e, consequentemente, uma tendência no resultado final do índice.

Como pressuposto, foram utilizados exclusivamente dados do censo demográfico do ano de 2010, do IBGE, utilizando setores censitários como unidade geográfica base para análises, o que possibilita uma série de vantagens na utilização, como monitorar a evolução das variáveis estudadas, devido à periodicidade e confiabilidade dos dados, permitindo comparações com informações de censos passados e com os futuros, além da descentralização de avaliação, permitindo identificar valores em unidades elementares (setores censitários).

Os indicadores foram compilados em planilhas derivadas dos arquivos originais do IBGE, mantendo os dados de interesse para cálculo, conforme apresentado na Tabela 1. A unificação do índice geral para cada setor censitário com base na equação 1. A seguir, foi criado um arquivo vetorial representando cada um desses indicadores por meio de integração da planilha de cálculo na tabela de atributos do arquivo vetorial de setores censitários do IBGE, combinados com os setores censitários do estado de Santa Catarina, definido como área de estudo (total de 11.882 setores, sendo 9.568 setores urbanos).

Por exemplo, o tema autonomia de renda possui os seguintes subtemas: distribuição de renda e propriedade domiciliar, que darão origem a indicadores IB01 e IB02, respectivamente. A metodologia de cálculo dos indicadores foi determinada com 0 pressuposto de gerar valores relativos, ou seja, variando entre 0 e 1 , buscando-se quantificar o quanto de cada medida corresponde ao universo total desta medida, onde, por exemplo, para se obter o indicador relacionado a mulheres responsáveis, o cálculo estabelece uma razão entre o total de mulheres chefes de família pelo total de chefes de família de determinado setor, permitindo obter uma razão percentual. Isso permite simplificar a análise e, ao mesmo tempo, manter a essência da informação buscada, fornecendo indicação do grau de deficiência do resultado prático do indicador por meio de relação percentual. 
Tabela 1 - Indicadores considerados para a composição do Índice Socioeconômico - ISE

\begin{tabular}{|c|c|c|c|c|}
\hline Tema & Subtema & Indicador & Cálculo & Cod. \\
\hline $\begin{array}{l}\frac{0}{0} \\
\frac{\pi}{0} \\
\frac{0}{5} \\
\frac{5}{w}\end{array}$ & $\begin{array}{l}\text { Mulheres } \\
\text { chefes de } \\
\text { família nos } \\
\text { domicílios }\end{array}$ & $\begin{array}{l}\text { Mulheres } \\
\text { responsáveis }\end{array}$ & $\begin{array}{l}\text { Mulheres chefe de família/Total de chefes de } \\
\text { família }\end{array}$ & IA01 \\
\hline \multirow{2}{*}{ 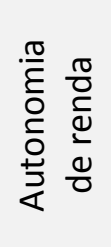 } & $\begin{array}{l}\text { Distribuição } \\
\text { de renda }\end{array}$ & $\begin{array}{l}\text { Responsáveis } 1 \\
\text { Salário Mínimos } \\
\text { (SM) }\end{array}$ & $\begin{array}{l}\text { Número de pessoas responsáveis por } \\
\text { domicílio com renda nominal menor que } 1 \\
\text { SM/Total de domicílios }\end{array}$ & IB01 \\
\hline & $\begin{array}{l}\text { Propriedade } \\
\text { domiciliar }\end{array}$ & $\begin{array}{l}\text { Domicílios não } \\
\text { próprios }\end{array}$ & $\begin{array}{l}\text { Domicílios permanentes não próprios/Total } \\
\text { de domicílios permanentes }\end{array}$ & IB02 \\
\hline \multirow{3}{*}{ 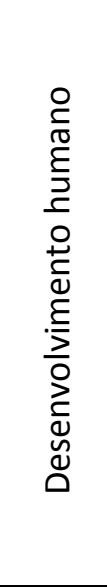 } & $\begin{array}{l}\text { Escolaridade } \\
\text { precária }\end{array}$ & $\begin{array}{l}\text { Responsáveis não } \\
\text { alfabetizados }\end{array}$ & $\begin{array}{l}\text { Pessoas responsáveis não alfabetizadas/ } \\
\text { Total de pessoas responsáveis }\end{array}$ & ICO1 \\
\hline & $\begin{array}{l}\text { Estimulo a } \\
\text { educação }\end{array}$ & $\begin{array}{l}\text { Crianças } \\
\text { alfabetizadas } \\
\text { precocemente } \\
\text { (5-9 anos) } \\
\text { Crianças } \\
\text { alfabetizadas } \\
\text { tardiamente } \\
\text { (10-14 anos) }\end{array}$ & $\begin{array}{l}\text { Pessoas alfabetizadas de } 10-14 \text { anos)/ } \\
\text { (total de pessoas com 10-14 anos) }\end{array}$ & ICO3 \\
\hline & Longevidade & $\begin{array}{l}\text { População com } 80 \\
\text { anos ou mais }\end{array}$ & $\begin{array}{l}\text { Pessoas com } 80 \text { anos ou mais/ } \\
\text { Total de Pessoas }\end{array}$ & ICO4 \\
\hline \multirow{7}{*}{ 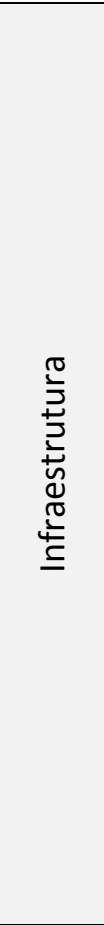 } & \multirow{3}{*}{$\begin{array}{l}\text { Saneamento } \\
\text { básico }\end{array}$} & $\begin{array}{l}\text { Precariedade de } \\
\text { abastecimento de } \\
\text { água }\end{array}$ & $\begin{array}{l}\text { Domicílios sem acesso à rede de água geral ou } \\
\text { poço na propriedade/Total de domicílios }\end{array}$ & ID01 \\
\hline & & $\begin{array}{l}\text { Precariedade } \\
\text { instalação sanitária }\end{array}$ & $\begin{array}{l}\text { Domicílios sem condições adequadas } \\
\text { sanitárias/Total de domicílios }\end{array}$ & ID02 \\
\hline & & $\begin{array}{l}\text { Precariedade coleta } \\
\text { de lixo }\end{array}$ & $\begin{array}{l}\text { Domicílios sem condições adequadas de } \\
\text { destinação de resíduos/Total de domicílios }\end{array}$ & ID03 \\
\hline & $\begin{array}{l}\text { Conforto } \\
\text { domiciliar }\end{array}$ & $\begin{array}{l}\text { Densidade } \\
\text { habitacional } \\
\text { (moradores/ } \\
\text { domicílios) }\end{array}$ & $\begin{array}{l}\text { Moradores de domicílios permanentes/ } \\
\text { Total de domicílios particulares }\end{array}$ & ID04 \\
\hline & Habitação & $\begin{array}{l}\text { Presença de } \\
\text { moradias } \\
\text { improvisadas }\end{array}$ & $\begin{array}{l}\text { Domicílios sem banheiro de uso exclusivo/ } \\
\text { Total de domicílios }\end{array}$ & ID05 \\
\hline & \multirow[b]{2}{*}{ Urbanização } & $\begin{array}{l}\text { Domicílios com } \\
\text { calçada }\end{array}$ & $\begin{array}{l}\text { Total de domicílios permanentes com } \\
\text { calçada/Total de domicílios permanentes }\end{array}$ & ID06 \\
\hline & & $\begin{array}{l}\text { Domicílios com } \\
\text { iluminação pública }\end{array}$ & $\begin{array}{l}\text { Total de domicílios particulares com } \\
\text { iluminação pública/Total de domicílios } \\
\text { permanentes }\end{array}$ & ID07 \\
\hline
\end{tabular}

Fonte: Adaptado de Sposati (1996; 2000), Genovez et al. (2001) e Silva Júnior (2007). 
Todos os indicadores foram calculados em termos relativos (razão entre parte de um total pelo total). Isso garante que o valor dos indicadores permaneça entre 0 e 1 , com exceção do indicador densidade domiciliar (moradores/domicílio). Para isso, foi necessária a normalização por meio de relação direta, considerando o maior valor de densidade domiciliar nos setores censitários do estado (sete pessoas/domicílio), e, dessa forma, após correção, quanto maior a densidade domiciliar mais próximo o seu valor de 1 , e, quanto menor, mais próximo de 0 .

A unificação do índice se deu por somatório dos valores normalizados de cada indicador, levando-se em conta o caráter qualitativo do indicador, ou seja, alguns indicadores possuem relação positiva com a promoção de melhores condições de vida, e outros, negativa. Como exemplo, cita-se a longevidade, indicador cujo índice tende a aumentar (positivamente) conforme a proporção de pessoas com 80 anos ou mais (ICO4), e, em contrapartida, quanto maior a proporção populacional com renda inferior a um salário mínimo (IB01), menor a condição socioeconômica no setor analisado. No caso do IC04, esse somará positivamente na contabilização, e o IB01 subtrairá. Com isso, a fórmula resulta em:

$$
\begin{aligned}
& I S E \\
& \quad=I A 01+I C 02+I C 04+I D 06+I D 07-I B 01-I B 02-I C 01-I C 03 \\
& \quad-I D 01-I D 02-I D 03-I D 04-I D 05
\end{aligned}
$$

Para a análise de resultados, foram considerados apenas os setores urbanos, haja vista que na zona rural, por possuir diferentes dinâmicas e acesso a infraestruturas da zona urbana, outros parâmetros deverão ser analisados para que se precise melhor a análise em setores rurais.

\section{Resultados e discussão}

O desenvolvimento dos índices para cada setor censitário permitiu a composição de mapa do Índice Socioeconômico (ISE) para o Estado de Santa Catarina, objeto de estudo de caso. A 
Figura 1 exemplifica o resultado final do mapeamento realizado. Devido à escala de trabalho ser em nível de setor censitário e abranger uma grande área de estudo, em nível de Estado, a apresentação do resultado em único mapa não possibilita o conhecimento adequado dos resultados, sendo recomendada a utilização de uma desagregação geográfica por município, especificamente para o perímetro urbano.

A amplitude do ISE calculado para os setores urbanos variou de $-4,77$ a 2,42 , sendo os menores valores correspondentes a áreas com piores condições socioeconômicas definidas no ambiente do ISE. Observa-se que a maioria dos setores apresentou valores positivos (54,5\% dos setores), indicando um balanço positivo entre o número de pessoas ou domicílios com condições socioeconômicas adequadas, ou seja, razão positiva entre famílias em condições adequadas (

Figura 2).

Figura 1 - Exemplo da distribuição geográfica do ISE no Estado de Santa Catarina 


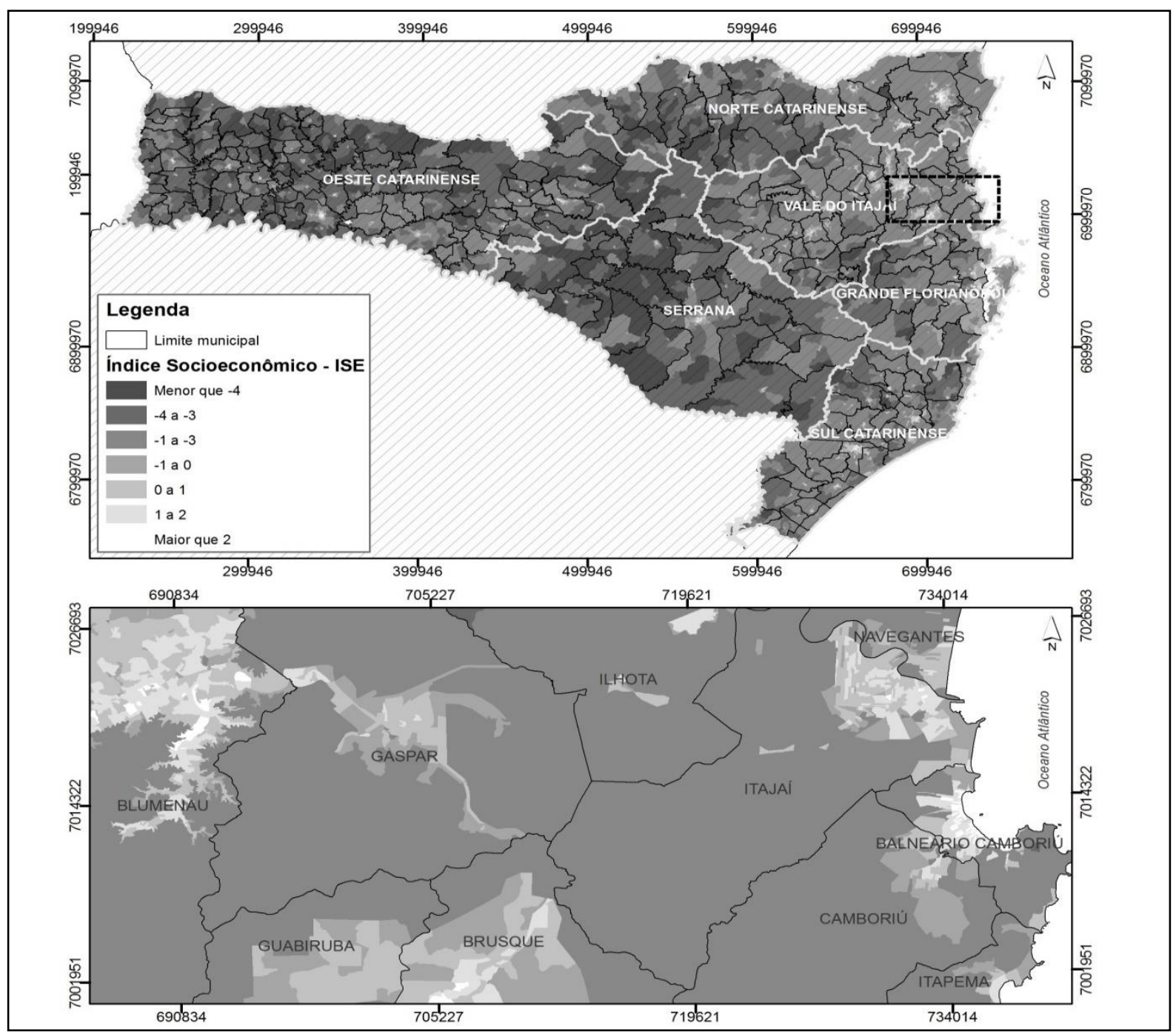

Fonte: Elaborado pelo autor com base em dados do IBGE.

Analisando o valor do ISE nos setores urbanos, foi evidenciado que a classe do ISE mais frequente foi a dos valores entre $0-1$, correspondendo a cerca de $37,4 \%$ dos setores analisados.

Figura 2 - Distribuição de frequência dos setores censitários urbanos analisados por classe de valor do Índice Socioeconômico - ISE 


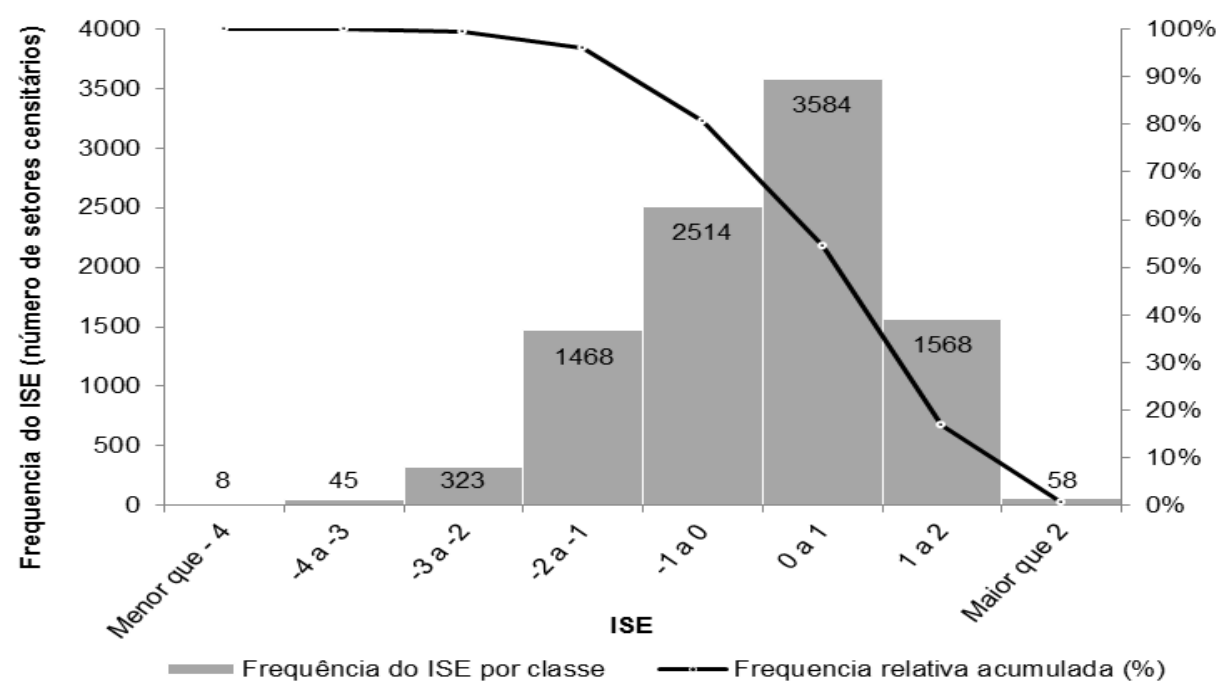

Fonte: Elaborado pelo autor com base em dados do IBGE.

Com relação ao desempenho do ISE entre as regiões do estado (mesorregiões), a Grande Florianópolis apresentou uma maior média geral de ISE $(0,317)$, seguida do Vale do Itajaí $(0,210)$, do Norte catarinense $(0,02)$, do Sul catarinense $(-0,14)$, da região Serrana ($0,31)$ e do Oeste catarinense $(-0,33)$. Comparando as regiões, foi observado que não há diferença significativa apenas entre as regiões Oeste e Serrana $(p=0,9994)$. Entre as regiões do Vale do Itajaí e Grande Florianópolis, há uma proximidade, porém não se pode afirmar a semelhança $(p=0,0307)$ (vide 
Figura 3). 
Figura 3 - Intervalo de confiança da comparação do ISE entre mesorregiões do Estado

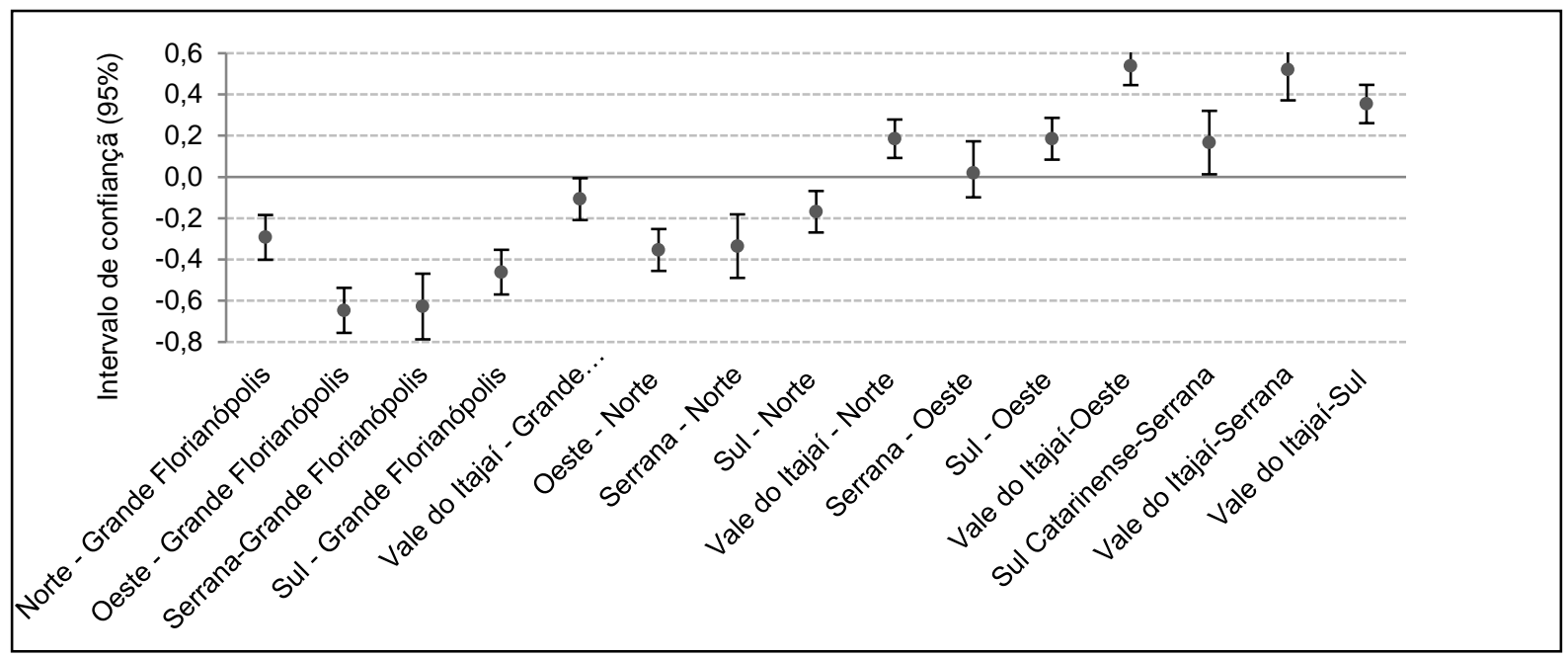

Fonte: Elaborado pelo autor com base em dados do IBGE.

Considerando os setores com as melhores e piores classificações por município, observa-se que as piores pontuações estão distribuídas por diversos municípios.

Figura 4 - Setores com melhores e menores valores do ISE por município de localização

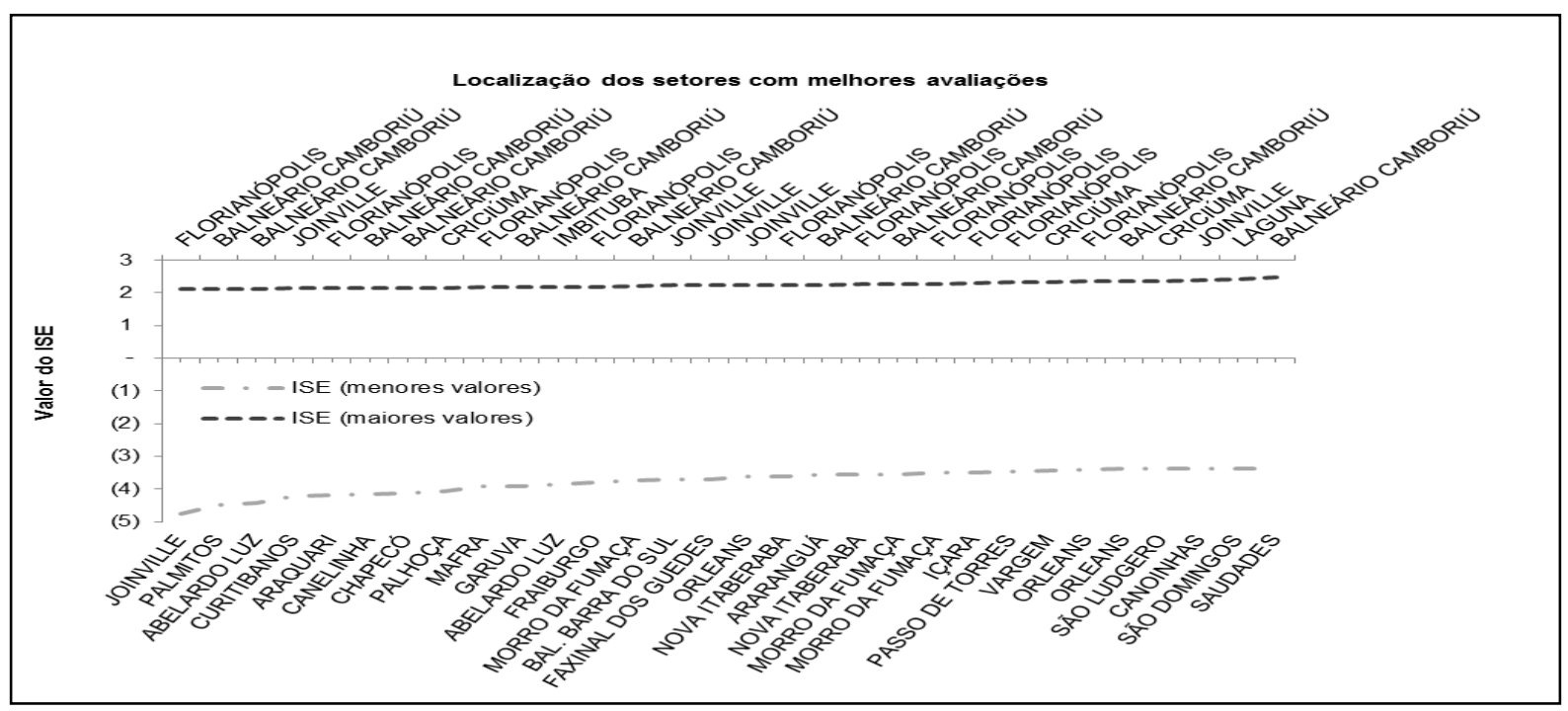

Fonte: Elaborado pelo autor com base em dados do IBGE.

Isso demonstrou uma grande desigualdade de grande parte dos municípios, destacando-se, por exemplo, Joinville, com o setor com a menor qualificação e, ao mesmo tempo, com setores com as melhores condições no Estado. Já valores mais altos foram 
concentrados em algumas cidades, com destaque para Balneário Camboriú, Joinville e Florianópolis.

\section{Considerações finais}

O objetivo da pesquisa foi atingido, sendo possível realizar uma avaliação exequível em grande escala, demandando poucos recursos financeiros e tempo de execução. A partir de outros estudos já realizados, foi possível aperfeiçoar a metodologia, sendo muito pertinente que trabalhos com este tema sejam fomentados e melhorados continuamente.

Recomenda-se a expansão do método para um nível nacional, além de melhorias na forma de apresentação do resultado em SIG para sistema relacionado a webgis, passível de interatividade por toda a sociedade.

Acredita-se que a utilização de indicadores possa contribuir para a melhoria das condições de vida da população, subsidiando o planejamento público e a tomada de decisão de gestores, além de permitir o acompanhamento e o controle social da própria população, que poderá monitorar o desenvolvimento de políticas públicas em seu município.

\section{Referências}

ALVES, M. T. G.; SOARES, J. F. Medidas de nível socioeconômico em pesquisas sociais: uma aplicação aos dados de uma pesquisa educacional. Opinião Pública, n. 15, v. 1, p. 1-30, 2009.

BAUER, R. A. Social Indicators. Cambridge/London: The M.I.T. Press, 1966.

BYRNE, D. Social Exclusion. Buckingham: Open University Press, 1999.

BARNES, M. Social Exclusion in Great Britain. An empirical investigation and comparison with the EU. Aldershot: Ashgate, 2005.

GENOVEZ, P. C. Território e desigualdades: análise espacial intra-urbana no estudo da dinâmica de exclusão/inclusão social no espaço urbano em São José dos Campos SP. Dissertação (Mestrado em Sensoriamento Remoto - Programa de Pós-Graduação em Sensoriamento Remoto) - Instituto Nacional de Pesquisas Espaciais, São José dos Campos, 2005. 
GENOVEZ, P. C. et al. Diagnóstico das Áreas de Exclusão/Inclusão Social através de Sistema de Informação Geográfica na Área Urbana de São José dos Campos - SP. Anais X SBSR. Foz do Iguaçu, 2001.

IBGE - Instituto Brasileiro de Geografia e Estatística. Base de informações do Censo Demográfico 2010: Resultados do Universo por setor censitário. Rio de Janeiro, 2011.

. Indicadores de desenvolvimento sustentável. Brasil: 2015 / IBGE, Coordenação de Recursos Naturais e Estudos Ambientais [e] Coordenação de Geografia. - Rio de Janeiro: IBGE, 2015a. 352p. (Estudos e pesquisas. Informação geográfica, ISSN 1517-1450; n. 10).

. Síntese de indicadores sociais: uma análise das condições de vida da população brasileira. 2015/ IBGE, Coordenação de População e Indicadores Sociais. - Rio de Janeiro: IBGE, 2015b. 137p. (Estudos e pesquisas. Informação demográfica e socioeconômica, ISSN 1516-3296; n. 35).

. Uma análise das condições de vida da população brasileira 2012. Síntese dos indicadores sociais. Disponível em:

<http://www.ibge.gov.br/home/estatistica/populacao/condicaodevida/indicadoresminimos /sinteseindicsociais2012/default.shtm>. Acesso em: 5 jul. 2017.

JANNUZZI, P. M. Indicadores sociais no Brasil. Conceitos fontes de dados e aplicações. 4. ed. Campinas: Alínea, 2009.

JICZM - Glossário. 2017. Journal of Integrated Coastal Zone Management. Disponível em: <http://www.aprh.pt/rgci/glossario/socioeconomico.html>. Acesso em: 7 jul. 2017.

LEMOS, J. J. S. Mapa da exclusão social no Brasil: radiografia de um país assimetricamente pobre. 2. ed. Fortaleza: Universidade Federal do Ceará, 2007.

MATHIESON, J. et al. Social Exclusion Meaning, measurement and experience and links to health inequalities a review of literature. WHO Social Exclusion Knowledge Network Background Paper 1, 2008.

NASCIMENTO, E.; MATIAS, L. F. Mapeamento da exclusão/inclusão social no espaço intraurbano com dados censitários: experiência a partir da cidade de Ponta Grossa (PR). Ponta Grossa: Terr@Plural, 2008.

SILVA JÚNIOR, W. Gestão pública e monitoramento Gestão pública e monitoramento das desilgualdades socioespaciais: o mapa da das desilgualdades socioespaciais: o mapa da a exclusão / inclusão social de Itajaí - SC. Ponta Grossa: Terr@Plural, 2007.

SINGER, Paul. Globalização e desemprego: diagnóstico e alternativas. 4. ed. São Paulo: Contexto, 2003. 
SPOSATI, A. Cidade, Território, Exclusão/Inclusão Social. Congresso Internacional de Geoinformação - GEO Brasil/2000, São Paulo. Disponível em:

<http://www.dpi.inpe.br/geopro/exclusao/cidade.pdf>. Acesso em: 7 jul. 2017.

. Mapa de Exclusão/Inclusão da Cidade de São Paulo. São Paulo: PUC-SP, 1996.

SOLIGO, V. Indicadores: conceito e complexidade do mensurar em estudos de fenômenos sociais. Est. Aval. Educ. São Paulo, v. 23, n. 52, p. 12-25, 2012.

TISCHER, V. Avaliação da aderência de indicadores socioambientais costeiros: estudo de caso do litoral de Santa Catarina. Revista Internacional de Ciências, Rio de Janeiro, v. 6, n. 2, p. 147-159, jul. 2016.

TISCHER, V.; POLETTE, M. Proposta metodológica de estabelecimento de indicadores socioambientais para a zona costeira brasileira. Revista Brasileira de Gestão e Desenvolvimento Regional. G\&DR. v. 12, n. 2, p. 355-373, Taubaté, 2016. 